ZU-TH 19/03

$\operatorname{IPPP} / 03 / 75$

$\mathrm{DCPT} / 03 / 150$

hep-ph/0311276

November 2003

\title{
Four-Particle Phase Space Integrals in Massless QCD
}

\author{
A. Gehrmann-De Ridder ${ }^{a}$, T. Gehrmann ${ }^{a}$ and G. Heinrich ${ }^{b}$ \\ ${ }^{a}$ Institut für Theoretische Physik, Universität Zürich, Winterthurerstrasse 190, CH-8057 Zürich, \\ Switzerland \\ ${ }^{b}$ Institute for Particle Physics Phenomenology, University of Durham, South Road, \\ Durham DH1 3LE, England
}

\begin{abstract}
The inclusive four-particle phase space integral of any $1 \rightarrow 4$ matrix element in massless QCD contains divergences due to the soft and collinear emission of up to two particles in the final state. We show that any term appearing in this phase space integral can be expressed as linear combination of only four master integrals. These four master integrals are all computed in dimensional regularisation up to their fourth order terms, relevant to next-to-next-to-leading order jet calculations, both in an analytic form and purely numerically. New analytical and numerical techniques are developed in this context. We introduce the tripole parametrisation of the four-parton phase space. Furthermore, we exploit unitarity relations between multi-parton phase space integrals and multi-loop integrals. For the numerical calculation, the iterated sector decomposition of loop integrals is extended to phase space integrals. The results in this paper lead to infrared subtraction terms needed for the double real radiation contributions to jet physics in $e^{+} e^{-}$annihilation at the next-to-next-to-leading order.
\end{abstract}




\section{Introduction}

In recent years, data on jet observables from LEP, HERA and the Tevatron have reached an impressive level of experimental precision. Using these data for a determination of the strong coupling constant, or as a measurement of parton distributions in the colliding hadrons, it turns out that the error on these extractions is largely dominated by the uncertainty on the underlying theoretical calculations, which are accurate only to the next-to-leading order (NLO) in perturbative QCD.

In order to improve on this situation, theoretical calculations of jet observables accurate to the nextto-next-to-leading order (NNLO) are mandatory. For each observable, they require several ingredients: To compute the corrections to an $n$-jet observable, one needs the two-loop $n$-parton matrix elements, the oneloop $(n+1)$-parton matrix elements and the tree level $(n+2)$-parton matrix elements. In the recent past, enormous progress has been made especially on the calculation of two-loop $2 \rightarrow 2$ and $1 \rightarrow 3$ QCD matrix elements, which are now known for all massless parton-parton scattering processes [1,2, 3, 4, relevant to hadron colliders as well as for $\gamma^{*} \rightarrow q \bar{q} g$ [5, 6] and its crossings [7]. Most of these results were even verified independently by different groups. For the corresponding partonic processes, the one-loop matrix elements with one additional parton and the tree-level matrix elements with two more partons are also known and form part of NLO programs for $1 \rightarrow 4[8$ ] and $2 \rightarrow 3$ reactions [9]. Since these matrix elements lead to infrared singularities due to one or two partons becoming theoretically unresolved (soft or collinear), one needs to find one- and two-particle subtraction terms, which account for these singularities in the matrix elements, and are sufficiently simple to be integrated analytically over the unresolved phase space. One-particle subtraction at tree level is well understood from NLO calculations [10,11, 12, and general algorithms are available for one-particle subtraction at one loop [13, 14, 15, in a form that could recently be integrated analytically [14,15. Tree-level two-particle subtraction terms have been extensively studied in the literature [16, 17, 18], their integration over the unresolved phase space could however up to now be performed only in one particular infrared subtraction scheme (the hybrid subtraction method) in the calculation of higher order corrections to the photon-plus-one-jet rate in $e^{+} e^{-}$annihilation [19] 20]. The same techniques (and the same scheme) were used very recently in the rederivation of the time-like gluonto-gluon splitting function from splitting amplitudes [21. A general two-particle subtraction procedure, consisting of subtraction terms which can be safely implemented numerically and at the same time are sufficiently simple to be integrated analytically is still lacking at the moment. It is the aim of the present paper to contribute to such a method by classifying and computing all four-particle phase space integrals relevant to $1 \rightarrow 4$ parton reactions in massless QCD.

One of the most widely used infrared subtraction schemes at NLO is the dipole formalism established by Catani and Seymour [12. The formalism relies on an exact factorisation of the $n$-particle phase space into an (n-1)-particle phase space and a so-called dipole phase space, which is, up to a normalisation factor, equal to a massless three-particle phase space. In this formalism, one derives (process-independent) dipole subtraction terms for all single unresolved partonic configurations, which are then integrated over the dipole phase space (corresponding to a three-parton configuration of emitter, unresolved parton and spectator). If combined appropriately, these integrals of the subtraction terms over the dipole phase space can be related to integrals of $1 \rightarrow 3$ tree level QCD matrix elements integrated over the three-particle phase space.

At NNLO, one encounters configurations of two unresolved partons between emitter and spectator. As will be shown below in Section 4.1 these can be accommodated into a tripole formulation, relying on the exact factorisation of the $n$-particle phase space into an $(n-2)$-particle phase space and a tripole phase space. This tripole phase space is proportional to the four-particle phase space. Based on this observation, one might envisage that all double unresolved partonic configurations which correspond to a single emitter-spectator pair may be expressed by appropriate tripole subtraction terms. To facilitate their analytic integration over the tripole phase space, these may be further combined to relate to integrals of $1 \rightarrow 4$ tree-level QCD matrix elements integrated over the four-particle phase space. In this paper, we show that all integrals of this type can be expressed as linear combinations of only four so-called master integrals, which we compute both analytically and numerically.

The reduction to master integrals follows largely methods developed for multi-loop integrals, and is briefly presented in Section 3. To compute the resulting master integrals analytically in Section 4 we develop first the tripole formulation of the four-particle phase space, which is then used to calculate two of 
the master integrals. We then show that the remaining two master integrals can be inferred from unitarity relations between known multi-loop integrals and phase space integrals. Further, one of the master integrals calculated explicitly, as well as a reducible integral, are rederived from unitarity relations as crosschecks.

In Section [5] the master integrals are calculated numerically. The infrared poles are isolated by an automated sector decomposition algorithm which already has proven useful in the calculation of multi-loop integrals 22 23], and which has been extended in 24] and in the present work to tackle infrared divergent integrals over a multi-parton phase space as well.

A summary and conclusions are given in Section [6. The Appendix contains formulae relevant to the massless $(1 \rightarrow n)$-parton phase space.

\section{Kinematics and Notation}

In this paper, we consider the decay of a massive particle $Q$ ( $Z$-boson, off-shell photon or Higgs-boson) into four massless QCD partons $\left(P_{1}, \ldots P_{4}: q \bar{q} g g, q \bar{q} q \bar{q}\right.$ or $\left.g g g g\right)$ :

$$
Q(q) \rightarrow P_{1}\left(p_{1}\right)+P_{2}\left(p_{2}\right)+P_{3}\left(p_{3}\right)+P_{4}\left(p_{4}\right),
$$

where $q, p_{1}, \ldots, p_{4}$ denote the momenta of the particles. The kinematics of this process are uniquely defined by the six Lorentz invariants $s_{i j}=2 p_{i} \cdot p_{j}$ formed by any pairs of partons. These appear in the numerator and denominator (propagators) of QCD matrix elements. Only five of them are independent, the sixth being fixed by energy-momentum conservation

$$
q^{2}=s_{12}+s_{13}+s_{14}+s_{23}+s_{24}+s_{34}
$$

Besides these, QCD matrix elements also contain propagators yielding triple invariants

$$
s_{i j k}=s_{i j}+s_{i k}+s_{j k} .
$$

Without loss of generality, we can always choose $P_{1}$ and $P_{2}$ such that the associated scalar product $s_{12}$ and triple invariants $s_{123}, s_{124}$ do not appear as a propagator in a given term of the squared matrix element.

Any single term in a squared four-particle QCD matrix element can then be expressed as

$$
T\left(S_{1}^{n_{1}} \ldots S_{q}^{n_{q}} ; D_{1}^{m_{1}} \ldots D_{t}^{m_{t}}\right)=\frac{S_{1}^{n_{1}} \ldots S_{q}^{n_{q}}}{D_{5}^{m_{1}} \ldots D_{t}^{m_{t}}}
$$

where the $S_{l}$ are the Lorentz invariants $s_{i j}$ and the $D_{l}$ are the propagators, being either invariants $s_{i j}$ or triple invariants $s_{i j k}$. The $n_{i}$ and $m_{i}$ are positive integers. It is assumed that whenever possible, scalar products in the numerator are expressed as linear combinations of the propagators, such that only a minimal number of different scalar products is present in the numerator. In fact, one finds that for $t-4$ different propagators, only $9-t$ different irreducible scalar products exist. The integral of the term (2.2) over the four-particle phase space is denoted by

$$
I_{T}\left(S_{1}^{n_{1}} \ldots S_{q}^{n_{q}} ; D_{5}^{m_{5}} \ldots D_{t}^{m_{t}}\right)=\int \mathrm{d} P S_{4} T\left(S_{1}^{n_{1}} \ldots S_{q}^{n_{q}} ; D_{5}^{m_{5}} \ldots D_{t}^{m_{t}}\right) .
$$

The phase space measure $\mathrm{d} P S_{4}$ is defined in A.5 in the appendix.

The topology of $I_{T}$ (interconnection of propagators and external momenta) is uniquely determined by the set of propagators $\left(D_{5}, \ldots, D_{t}\right)$. In order to visualise the topology of $I_{T}$ in the form of a cut diagram, we use the Cutkosky rules [25, 26] to introduce the four cut-propagators

$$
\frac{1}{D_{i}}=2 \pi i \delta^{+}\left(p_{i}^{2}\right)=\frac{1}{p_{i}^{2}+i 0}-\frac{1}{p_{i}^{2}-i 0} \quad(i=1, \ldots 4) .
$$

With the help of the cut-propagators, the four-particle phase space measure in (2.3) can be written as

$$
\mathrm{d} P S_{4}=i^{-4} \frac{\mathrm{d}^{d} p_{1}}{(2 \pi)^{d}} \frac{\mathrm{d}^{d} p_{2}}{(2 \pi)^{d}} \frac{\mathrm{d}^{d} p_{3}}{(2 \pi)^{d}} \frac{\mathrm{d}^{d} p_{4}}{(2 \pi)^{d}}(2 \pi)^{d} \delta^{d}\left(q-p_{1}-p_{2}-p_{3}-p_{4}\right) \frac{1}{D_{1} D_{2} D_{3} D_{4}}
$$


such that the integral $I_{T}$ can be expressed as a cut through a three-loop vacuum polarisation diagram. Any integral $I_{T}$ for a given topology (fixed set of $t-4$ propagators) can be attributed to a class of integrals $I_{T(t, r, s)}$ of identical numerator mass dimension $s=\sum_{i=1}^{9-t} n_{i}$ and denominator mass dimension $r=\sum_{i=1}^{t} m_{i}$. It should be noted that we also allow the cut-propagators to appear in general with integer powers larger than unity, without further specifying the physical meaning of those. From combinatorics, one finds that the class $I_{T(t, r, s)}$ contains

$$
N_{t, r, s}=\left(\begin{array}{l}
r-1 \\
r-t
\end{array}\right)\left(\begin{array}{c}
8-t+s \\
s
\end{array}\right)
$$

different integrals.

\section{Reduction to Master Integrals}

The number $N\left(I_{t, r, s}\right)$ of the integrals grows quickly as $r, s$ increase, but the integrals are not all linearly independent. These linear relations are the so-called integration-by-part (IBP) identities, which were originally formulated for multi-loop two-point functions [27, 28 with ordinary loop propagators, but can be safely extended to multi-loop integrals with cut propagators [26]. These identities follow from the fact that the integral over the total derivative with respect to any loop momentum vanishes in dimensional regularisation

$$
\int \frac{\mathrm{d}^{d} k}{(2 \pi)^{d}} \frac{\partial}{\partial k^{\mu}} J(k, \ldots)=0,
$$

where $J$ is any combination of propagators, scalar products and loop momentum vectors. $J$ can be a vector or tensor of any rank. The IBP identities for three-loop two-point functions were derived and solved symbolically a long time ago 28 in the context of the calculation of the three-loop $\beta$-function. The solution of these identities was implemented into the program package Mincer [29] written in the algebraic programming language Form 30. Mincer was widely used in recent years to compute a large number of multi-loop corrections which correspond to three-loop two-point functions or can be related to them by expansion. Reviews of these results can be found in 31,32 .

In recent times, IBP-type relations between multi-loop integrals with more than two legs have been studied extensively. In particular, it was found that scalar multi-loop integrals with three or more legs fulfil, besides the ordinary IBP relations, also relations following from the invariance of the integrals under an infinitesimal Lorentz transformation. These Lorentz invariance (LI) identities, combined with the IBP identities, were used extensively in the computation of two-loop four-point matrix elements [1, 2, 3, 4, 5, 6]. In a related development, it was observed that the IBP identities for Feynman integrals with the same total number of external and loop momenta are equivalent [33, a development that was used soon thereafter to compute two-loop three-point functions 34.

Exploiting the observation that differentiations involved in the integration-by-parts identities are insensitive to the imaginary parts of the two terms in a cut-propagator 26], one can generalise the IBP method to phase space integrals over tree-level or loop matrix elements. In the solution of the IBP equations for a given cut integral, one obtains integrals with one or more of the cut propagators eliminated. These integrals can be discarded, since they do not contribute anymore to the same cut of the underlying multi-loop diagram.

To derive the reduction of any given integral $I_{T}$ of the form (2.3), we use the same strategy 35. as applied in the reduction of the two-loop four-point functions in 36 by generating all IBP identities for integrals up to the maximum values of $r$ and $s$ required for the integration of QCD four-particle matrix elements. From dimensional counting, one finds $r \leq 8, s \leq 3$. The solution algorithm [35] proceeds by solving all identities up to these values using the computer algebra languages Form 30. and Maple [37.

After carrying out the reduction for all topologies that can be formed from the invariants and triple invariants involving the four final state momenta, we find that all inclusive four-particle phase space integrals of massless QCD matrix elements can be expressed as a linear combination of four master integrals. These 
four master integrals are:

$$
\begin{aligned}
R_{4} & \equiv=\int \mathrm{d} P S_{4}=P_{4} \\
R_{8, a} & \equiv=\int \mathrm{d} P S_{4} \frac{1}{s_{134} s_{234}}
\end{aligned}
$$

The reduction procedure generates coefficients containing explicit poles of up to $1 / \epsilon^{4}$ in front of $R_{4}$ and up to $1 / \epsilon^{2}$ in front of $R_{6}$, while the coefficients multiplying $R_{8, a}$ and $R_{8, b}$ are finite.

In the following two sections, we will compute these four master integrals both analytically and numerically to a sufficiently high order in $\epsilon$ required for the calculation of integrated four-particle QCD matrix elements.

\section{Analytic Calculation of Master Integrals}

\subsection{Tripole Phase Space}

The dipole formalism [12] of Catani and Seymour is a subtraction formalism for the calculation of jet cross sections at NLO. The main features of this formalism are factorisation formulae for matrix elements and phase space which locally reproduce single soft and single collinear behaviour of the cross sections in the appropriate limits. The corresponding dipole contributions to the cross sections can be integrated analytically and numerically over the whole phase space. In particular, the exact factorisation of the threeparton phase space into a dipole phase space and a two-parton phase space is obtained by redefining a set of three massless on-shell momenta (emitter, unresolved parton, spectator) into two on-shell massless momenta. This procedure was further generalised by Kosower [17, also accounting for the recombination of more than three partonic momenta. In 17] a non-linear remapping of momenta, which smoothly interpolates between all potentially singular regions (as required for the numerical implementation) was introduced. Such a non-linear remapping is however inappropriate for analytic integration, and we shall derive a linear remapping below.

As an extension of [12, we introduce a parametrisation of momenta allowing the factorisation of a massless $n$-parton phase space into a massless $(n-2)$-parton phase space times a tripole phase space factor:

$$
\mathrm{d} P S_{n}\left(p_{1}, \ldots, p_{n}\right)=\mathrm{d} P S_{n-2}\left(p_{1}, \ldots, p_{n-4}, \tilde{p}_{n-3}, \tilde{p}_{n-2}\right) \mathrm{d} P S_{T}
$$

In particular, it enables to factorise exactly the phase space of four on-shell parton momenta $\left(p_{1} . . p_{4}\right)$, which can also denote the four-particle contribution to an $(n>4)$-particle phase space, into a two-parton phase space times a tripole phase space factor

$$
\mathrm{d} P S_{4}\left(p_{1}, \ldots, p_{4}\right)=\mathrm{d} P S_{2}\left(p_{\widetilde{134}}, p_{\tilde{2}}\right) \mathrm{d} P S_{T} .
$$

In this parametrisation, $p_{1}$ is the momentum of the emitter, $p_{2}$ the one of the spectator, while $p_{3}$ and $p_{4}$ are the momenta of the unresolved partons. by

The tripole momenta $\left(p_{\widetilde{134}}, p_{\tilde{2}}\right)$ are combinations of four on-shell parton momenta. They are defined

$$
\begin{aligned}
p_{\widetilde{134}}^{\mu} & =p_{1}^{\mu}+p_{3}^{\mu}+p_{4}^{\mu}-\frac{y_{134,2}}{1-y_{134,2}} p_{2}^{\mu} \\
p_{\tilde{2}}^{\mu} & =\frac{1}{1-y_{134,2}} p_{2}^{\mu},
\end{aligned}
$$


where the dimensionless variable $y_{134,2}$ is

$$
y_{134,2}=\frac{p_{1} \cdot p_{3}+p_{1} \cdot p_{4}+p_{3} \cdot p_{4}}{p_{1} \cdot p_{3}+p_{1} \cdot p_{4}+p_{3} \cdot p_{4}+p_{1} \cdot p_{2}+p_{2} \cdot p_{3}+p_{2} \cdot p_{4}} .
$$

The tripole momenta are on-shell $\left(p_{\widetilde{134}}^{2}=p_{\tilde{2}}^{2}=0\right)$ and momentum conservation is implemented exactly

$$
p_{1}^{\mu}+p_{2}^{\mu}+p_{3}^{\mu}+p_{4}^{\mu}=p_{134}^{\mu}+p_{\tilde{2}}^{\mu} .
$$

To derive the tripole factorisation of $\mathrm{d} P S_{4}$, we start from its definition in terms of the invariants $s_{i j}$

$$
\begin{aligned}
\mathrm{d} P S_{4}= & (2 \pi)^{4-3 d}\left(q^{2}\right)^{3-\frac{d}{2}} 2^{1-2 d}\left(-\Delta_{4}\right)^{\frac{d-5}{2}} \Theta\left(-\Delta_{4}\right) \delta\left(s_{12}+s_{13}+s_{14}+s_{23}+s_{24}+s_{34}-q^{2}\right) \\
& \int \mathrm{d} \Omega_{d-1} \mathrm{~d} \Omega_{d-2} \mathrm{~d} \Omega_{d-3} \mathrm{~d} s_{12} \mathrm{~d} s_{13} \mathrm{~d} s_{14} \mathrm{~d} s_{23} \mathrm{~d} s_{24} \mathrm{~d} s_{34},
\end{aligned}
$$

where the four-particle Gram determinant $\Delta_{4}$ is given by

$$
\begin{aligned}
+\Delta_{4}= & {\left[s_{12}^{2} s_{34}^{2}+s_{13}^{2} s_{24}^{2}+s_{14}{ }^{2} s_{23}{ }^{2}\right.} \\
& \left.-2\left(s_{12} s_{23} s_{34} s_{14}+s_{13} s_{23} s_{24} s_{14}+s_{12} s_{24} s_{34} s_{13}\right)\right] \\
= & \lambda\left(s_{12} s_{34}, s_{13} s_{24}, s_{14} s_{23}\right)
\end{aligned}
$$

and $\lambda$ is the Källen function, $\lambda(x, y, z)=x^{2}+y^{2}+z^{2}-2(x y+x z+y z)$.

In order to obtain the massless four-parton phase space $\mathrm{d} P S_{4}$ in terms of dimensionless invariants defined with $p_{\widetilde{134}}, p_{\tilde{2}}$ as given in (4.2), the invariants $s_{i j}$ need to be substituted as follows

$$
\begin{aligned}
& s_{23}=\left(1-y_{134,2}\right) s_{\tilde{2} 3}=\left(1-y_{134}\right) q^{2} z_{1}, \\
& s_{24}=\left(1-y_{134,2}\right) s_{\tilde{2} 4}=\left(1-y_{134}\right) q^{2} z_{2}, \\
& s_{12}=\left(1-y_{134,2}\right) s_{12}=\left(1-y_{134}\right) q^{2}\left(1-z_{1}-z_{2}\right) .
\end{aligned}
$$

The last simplification occurs since, in the case of only four partons in the final state, one has

$$
2 p_{\widetilde{134}} \cdot p_{\tilde{2}}=s_{\widetilde{1234}}=s_{1234}=q^{2}
$$

and $y_{134,2}$ is the dimensionless variable $y_{134}=s_{134} / q^{2}$. The fractions of momenta $z_{i}$ are defined by:

$$
\begin{aligned}
& z_{1}=\frac{p_{3} \cdot p_{\tilde{2}}}{p_{\widetilde{134}} \cdot p_{\tilde{2}}}, \\
& z_{2}=\frac{p_{4} \cdot p_{\tilde{2}}}{p_{\widetilde{134}} \cdot p_{\tilde{2}}} .
\end{aligned}
$$

In the tripole phase space $\mathrm{d} P S_{T}$, the variables $s_{13}, s_{14}$ and $s_{134}$ remain unchanged and the triple invariant $s_{134}$ is used instead of $s_{34}$.

From the four-parton phase space, one can easily factorise a two-parton phase space factor $\mathrm{d} P S_{2}$ as defined in the Appendix using (4.7). The integration variables in $\mathrm{d} P S_{4}$ become

$$
\mathrm{d} s_{12} \mathrm{~d} s_{13} \mathrm{~d} s_{14} \mathrm{~d} s_{23} \mathrm{~d} s_{24} \mathrm{~d} s_{134}=\left(1-y_{134}\right)^{2}\left(q^{2}\right)^{5} \mathrm{~d} s_{1234} \mathrm{~d} y_{13} \mathrm{~d} y_{14} \mathrm{~d} z_{1} \mathrm{~d} z_{2} \mathrm{~d} y_{134},
$$

where the $\mathrm{d} s_{1234}$ on the right-hand side becomes part of the two-parton phase space while the other integration variables contribute to the tripole phase space factor $\mathrm{d} P S_{T}$. Furthermore, the Gram determinant $\Delta_{4}$ is rescaled as

$$
\Delta_{4}=\left(1-y_{134}\right)^{2}\left(q^{2}\right)^{4} \Delta_{4}^{\prime},
$$

with

$$
\Delta_{4}^{\prime}=\lambda\left(y_{12}\left(y_{134}-y_{13}-y_{14}\right), y_{13} z_{2}, y_{14} z_{1}\right)
$$


The factorised form of the four-parton phase space yielding the tripole phase space factor then reads:

$$
\mathrm{d} P S_{4}=\mathrm{d} P S_{2} \mathrm{~d} P S_{T},
$$

with

$$
\mathrm{d} P S_{T}=\left(q^{2}\right)^{2-2 \epsilon} \frac{2^{-10} \pi^{-5+2 \epsilon}}{\Gamma(1-2 \epsilon)}\left(1-y_{134}\right)^{1-2 \epsilon}\left(-\Delta_{4}^{\prime}\right)^{-1 / 2-\epsilon} \Theta\left(-\Delta_{4}^{\prime}\right) \mathrm{d} y_{13} \mathrm{~d} y_{14} \mathrm{~d} z_{1} \mathrm{~d} z_{2} \mathrm{~d} y_{134} .
$$

This tripole phase space factor $\mathrm{d} P S_{T}$ is proportional to the four-parton phase space, since the twoparton phase space is a constant. It is also proportional to the triple collinear phase space factor given in 20] obtained by requiring a special kinematical situation in which the three partons whose momenta are $p_{1}, p_{3}, p_{4}$ are collinear. In [20] this triple collinear phase space was used to compute the integral over the subtraction term appropriate to this kinematics, the triple collinear splitting function. The normalisation factor relating triple collinear phase space and tripole phase space is just $\left(1-y_{134}\right)^{1-2 \epsilon}$. It follows clearly that in the triple collinear limit where $y_{134} \rightarrow 0$, the tripole phase space factorisation is appropriate.

The volume of the four-parton phase space is well-known. However, we shall now rederive it using the tripole parametrisation described above, to demonstrate an application of the factorisation formula (4.10) and to illustrate the necessary variable transformations.

In this formula, $-\Delta_{4}^{\prime}$ can be written as a quadratic in $y_{13}$, the first variable to be integrated over. We obtain

$$
-\Delta_{4}^{\prime}=\left(1-z_{1}\right)^{2}\left(y_{13}-y_{13, a}\right)\left(y_{13, b}-y_{13}\right),
$$

with $y_{13, a}, y_{13, b}$ being the roots of the quadratic. Using

$$
y_{13}=\left(y_{13, b}-y_{13, a}\right) \chi+y_{13, a},
$$

one has

$$
\int\left(-\Delta_{4}^{\prime}\right)^{-1 / 2-\epsilon} \mathrm{d} y_{13}=\left(1-z_{1}\right)^{-1-2 \epsilon}\left(y_{13, b}-y_{13, a}\right)^{-2 \epsilon} \int_{0}^{1} \mathrm{~d} \chi[\chi(1-\chi)]^{-1 / 2-\epsilon} .
$$

The root difference $\left(y_{13, b}-y_{13, a}\right)$ satisfies the relation

$$
\left(1-z_{1}\right)^{4}\left(y_{13, b}-y_{13, a}\right)^{2}=16 z_{1} z_{2} y_{14}\left(1-z_{1}-z_{2}\right)\left(y_{134}\left(1-z_{1}\right)-y_{14}\right) \equiv \delta_{y_{13}} .
$$

Requiring that the Gram determinant $-\Delta_{4}^{\prime}$ is positive suggests the following change of variables

$$
\begin{aligned}
y_{14} & =y_{134}\left(1-z_{1}\right) v, \\
z_{2} & =\left(1-z_{1}\right) t .
\end{aligned}
$$

with $t, v$ ranging between 0 and 1 . Then $\delta_{y_{13}}$ completely factorises in these new variables:

$$
\delta_{y_{13}}=16 y_{134}^{2}\left(1-z_{1}\right)^{4} z_{1} t(1-t) v(1-v),
$$

such that the volume of the four-parton phase space then becomes:

$$
\begin{aligned}
\int \mathrm{d} P S_{4}= & \int \mathrm{d} P S_{2}\left(q^{2}\right)^{2-2 \epsilon} 2^{-10} \pi^{-5+2 \epsilon} \frac{1}{\Gamma(1-2 \epsilon)} \\
& \frac{\Gamma^{2}(1 / 2-\epsilon)}{\Gamma(1-2 \epsilon)} \int_{0}^{1} \mathrm{~d} y_{134}\left(1-y_{134}\right)^{1-2 \epsilon} y_{134}^{1-2 \epsilon} \int_{0}^{1} \mathrm{~d} z_{1}\left(1-z_{1}\right)^{1-2 \epsilon} z_{1}^{-\epsilon} \\
& \int_{0}^{1} \mathrm{~d} t t^{-\epsilon}(1-t)^{-\epsilon} \int_{0}^{1} \mathrm{~d} v v^{-\epsilon}(1-v)^{-\epsilon} .
\end{aligned}
$$

The integrations can be performed straightforwardly. Finally the volume of the four-parton phase space reads:

$$
R_{4}=P_{4}=\int \mathrm{d} P S_{4}=P_{2} \frac{(4 \pi)^{2 \epsilon}}{2^{8} \pi^{4}}\left(q^{2}\right)^{2-2 \epsilon} \frac{\Gamma^{3}(1-\epsilon) \Gamma(2-2 \epsilon)}{\Gamma(3-3 \epsilon) \Gamma(4-4 \epsilon)},
$$


where the volume of the two-particle phase space $P_{2}$ (A.9) has been factored out. Introducing the common normalisation factor

$$
S_{\Gamma}=P_{2}\left(\frac{(4 \pi)^{\epsilon}}{16 \pi^{2} \Gamma(1-\epsilon)}\right)^{2},
$$

one arrives at

$$
\begin{aligned}
R_{4}=S_{\Gamma}\left(q^{2}\right)^{2-2 \epsilon} & \frac{\Gamma^{5}(1-\epsilon) \Gamma(2-2 \epsilon)}{\Gamma(3-3 \epsilon) \Gamma(4-4 \epsilon)} \\
=S_{\Gamma}\left(q^{2}\right)^{2-2 \epsilon} & {\left[\frac{1}{12}+\frac{59}{72} \epsilon+\epsilon^{2}\left(\frac{2263}{432}-\frac{\pi^{2}}{9}\right)+\epsilon^{3}\left(\frac{72023}{2592}-\frac{59 \pi^{2}}{54}-\frac{13 \zeta_{3}}{6}\right)\right.} \\
& \left.+\epsilon^{4}\left(\frac{2073631}{15552}-\frac{2263 \pi^{2}}{324}-\frac{767 \zeta_{3}}{36}+\frac{\pi^{4}}{1080}\right)+\mathcal{O}\left(\epsilon^{5}\right)\right] .
\end{aligned}
$$

\subsection{Calculation of $R_{8, a}$}

Using the factorisation of $\mathrm{d} P S_{4}$ into the tripole phase space $\mathrm{d} P S_{T}$ and $P_{2}=\int \mathrm{d} P S_{2}$, the master integral $R_{8, a}$ defined by

$$
R_{8, a}=\int \mathrm{d} P S_{4} \frac{1}{s_{13} s_{14} s_{23} s_{24}}
$$

can be written as follows:

$$
\begin{aligned}
R_{8, a}= & P_{2} \int \mathrm{d} P S_{T}\left(1-y_{134}\right)^{-2}\left(q^{2}\right)^{-4} \frac{1}{y_{13} y_{14} z_{1} z_{2}} \\
= & P_{2}\left(q^{2}\right)^{-2-2 \epsilon} 2^{-10} \pi^{-5+2 \epsilon} \frac{1}{\Gamma(1-2 \epsilon)} \\
& \int\left(1-y_{134}\right)^{-1-2 \epsilon}\left(-\Delta_{4}^{\prime}\right)^{-1 / 2-\epsilon} \Theta\left(-\Delta_{4}^{\prime}\right) \mathrm{d} y_{13} \mathrm{~d} y_{14} \mathrm{~d} z_{1} \mathrm{~d} z_{2} \mathrm{~d} y_{134} \frac{1}{y_{13} y_{14} z_{1} z_{2}} .
\end{aligned}
$$

To perform the integrations, one factorises the Gram determinant using the same change of variables as for the evaluation of $R_{4}$. However due to the presence of the invariants in the denominator of the

integrands, the integrations are more complicated. Indeed, hypergeometric functions of involved rational arguments occur at different stages of these five subsequent analytic integrations. Using several non-linear transformations [38, 39] on these functions where appropriate, all integrals can be carried out in a closed analytic form, yielding finally

$$
\begin{aligned}
R_{8, a}=S_{\Gamma}\left(q^{2}\right)^{-2-2 \epsilon} & {\left[\frac{6}{\epsilon^{4}} \frac{\Gamma^{5}(1-\epsilon) \Gamma(1-2 \epsilon)}{\Gamma(1-3 \epsilon) \Gamma(1-4 \epsilon)}{ }_{4} F_{3}(1,-\epsilon,-\epsilon,-\epsilon ;-3 \epsilon, 1-\epsilon, 1+\epsilon ; 1)\right.} \\
& \left.-\frac{1}{\epsilon^{4}} \frac{\Gamma^{3}(1-\epsilon) \Gamma(1+\epsilon) \Gamma^{3}(1-2 \epsilon)}{\Gamma^{2}(1-4 \epsilon)}{ }_{3} F_{2}(-2 \epsilon,-2 \epsilon,-2 \epsilon ;-4 \epsilon, 1-2 \epsilon ; 1)\right] .
\end{aligned}
$$

Expanded up to finite order in $\epsilon, R_{8, a}$ reads

$$
R_{8, a}=S_{\Gamma}\left(q^{2}\right)^{-2-2 \epsilon}\left[\frac{5}{\epsilon^{4}}-\frac{20 \pi^{2}}{3 \epsilon^{2}}-\frac{126 \zeta_{3}}{\epsilon}+\frac{7 \pi^{4}}{18}+\mathcal{O}(\epsilon)\right] .
$$

\subsection{Unitarity Relation and Calculation of $R_{6}$ and $R_{8, b}$}

The use of unitarity to obtain relations between loop integrals and phase space integrals can be found in various contexts in the literature. In [40, unitarity is exploited to compute one-loop integrals from known single unresolved phase space integrals. To do the inverse, i.e. to derive phase space integrals from multi-loop integrals, has been exploited for example in [41. In [42, it was moreover attempted to 
implement the unitarity cancellation of infrared singularities to compute jet observables purely numerically as appropriately weighted loop integrals.

As outlined in Section 2 one can view the phase space integrals as particular cuts of multi-loop diagrams. In the case considered here, the four-particle phase space master integrals are particular cuts of massless three-loop two-point functions, which were studied extensively in the literature [43, 28, 29]. In particular, all master integrals appearing in these three-loop two-point functions are either known exactly or to a very high order in $\epsilon$. These three master integrals are 43 28]:

$$
\begin{aligned}
& I_{4}=\bigodot=\frac{(4 \pi)^{3 \epsilon}}{\left(16 \pi^{2}\right)^{3}} \frac{\Gamma(1+3 \epsilon) \Gamma(1-3 \epsilon) \Gamma^{4}(1-\epsilon)}{3 \epsilon \Gamma(3-3 \epsilon) \Gamma(4-4 \epsilon)}\left(-q^{2}\right)^{2-3 \epsilon}, \\
& I_{6}=-\left(12=\frac{(4 \pi)^{3 \epsilon}}{\left(16 \pi^{2}\right)^{3}} \frac{\Gamma^{6}(1-\epsilon) \Gamma^{3}(1+\epsilon)}{3 \epsilon^{3} \Gamma^{3}(2-2 \epsilon)}\left[1+\epsilon+\epsilon^{2}+\epsilon^{3}\left(14 \zeta_{3}-7\right)\right.\right. \\
& \left.+\epsilon^{4}\left(-67+14 \zeta_{3}+\frac{21 \pi^{4}}{90}\right)+\mathcal{O}\left(\epsilon^{5}\right)\right]\left(-q^{2}\right)^{-3 \epsilon}
\end{aligned}
$$

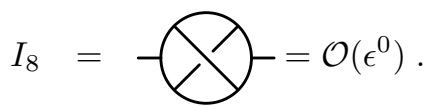

The optical theorem relates the imaginary part of a loop diagram to the sum over all its cuts in the form of a unitarity relation:
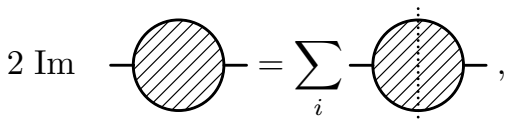

where $i$ enumerates all possible ways to cut the vacuum polarisation diagram into two connected amplitudes under the condition that the final states resulting from the cut lines belong to physical processes. It has to be kept in mind that this equation relates matrix elements, not just loop and phase space integrals. In the case of the master integrals considered here, one obtains the corresponding matrix elements by considering a scalar theory involving scalar three- and four-point vertices and two-point as well as four-point couplings to an external scalar current. Accordingly, one has to include a factor $i$ for each propagator and a factor $-i$ for each vertex to obtain the desired relations among master integrals.

If all but one of the cuts are known, the unitarity relation can be used to infer the remaining unknown cut. In the case considered here, the three-loop two-point functions can have

1. two-particle cuts: two-loop vertex function or product of two one-loop vertex functions integrated over two-particle phase space.

2. three-particle cuts: one-loop four-point function integrated over three-particle phase space.

3. four-particle cuts: tree-level matrix element integrated over four-parton phase space.

As we shall see below, the functions appearing in the two-particle cuts are well known from other calculations, and the three-particle cuts can be computed more easily than the four-particle cuts. The unitarity relation following from the optical theorem can therefore be used to determine the remaining four-particle phase space master integrals $R_{6}$ and $R_{8, b}$. Before computing these, we illustrate the application of the method on the rederivation of $R_{4}$.

The unitarity relation relevant to $R_{4}$ reads pictorially:

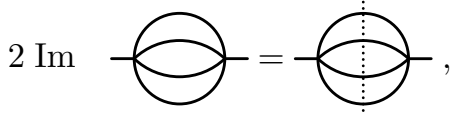

which yields

$$
2 \operatorname{Im} I_{4}=R_{4} .
$$


Using the relation

$$
\operatorname{Im}\left((-1)^{-n \epsilon} \Gamma(1+n \epsilon) \Gamma(1-n \epsilon) \frac{1}{n \epsilon}\right)=\pi
$$

which is valid to all orders in $\epsilon$, one can extract $R_{4}$ from the value of $I_{4}$ listed in (4.19):

$$
R_{4}=S_{\Gamma}\left(q^{2}\right)^{2-2 \epsilon} \frac{\Gamma^{5}(1-\epsilon) \Gamma(2-2 \epsilon)}{\Gamma(3-3 \epsilon) \Gamma(4-4 \epsilon)}
$$

in accordance with the result obtained by explicit computation in (4.14) above.

To determine $R_{6}$, we use the unitarity relation obtained for $I_{6}$ :

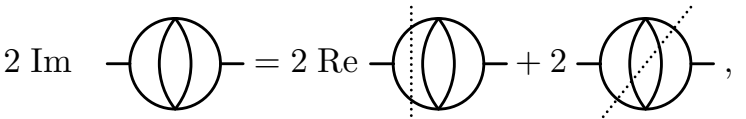

reading

$$
2 \operatorname{Im} I_{6}=-2 P_{2} \operatorname{Re} A_{4}+2 R_{6} .
$$

The two-loop vertex integral $A_{4}$ is known from form-factor calculations [44, and appeared also in the reduction of on-shell and off-shell massless two-loop four-point functions [45]:

$$
\begin{aligned}
A_{4}=\left(\frac{(4 \pi)^{\epsilon}}{16 \pi^{2}} \frac{\Gamma(1+\epsilon) \Gamma^{2}(1-\epsilon)}{\Gamma(1-2 \epsilon)}\right)^{2}\left(-q^{2}\right)^{-2 \epsilon} & {\left[-\frac{1}{2 \epsilon^{2}}-\frac{5}{2 \epsilon}-\left(\frac{19}{2}+\frac{\pi^{2}}{6}\right)-\left(\frac{65}{2}+\frac{5 \pi^{2}}{6}-2 \zeta_{3}\right) \epsilon\right.} \\
& \left.-\left(\frac{211}{2}+\frac{19 \pi^{2}}{6}-10 \zeta_{3}\right) \epsilon^{2}+\mathcal{O}\left(\epsilon^{3}\right)\right] .
\end{aligned}
$$

Therefore, $R_{6}$ can be immediately read off from (4.24) to be

$$
\begin{aligned}
R_{6}=S_{\Gamma}\left(q^{2}\right)^{-2 \epsilon}[ & -1+\frac{\pi^{2}}{6}+\epsilon\left(-12+\frac{5 \pi^{2}}{6}+9 \zeta_{3}\right) \\
& \left.+\epsilon^{2}\left(-91+\frac{9 \pi^{2}}{2}+45 \zeta_{3}+\frac{61 \pi^{4}}{180}\right)+\mathcal{O}\left(\epsilon^{3}\right)\right] .
\end{aligned}
$$

In order to obtain $R_{8, b}$, we employ

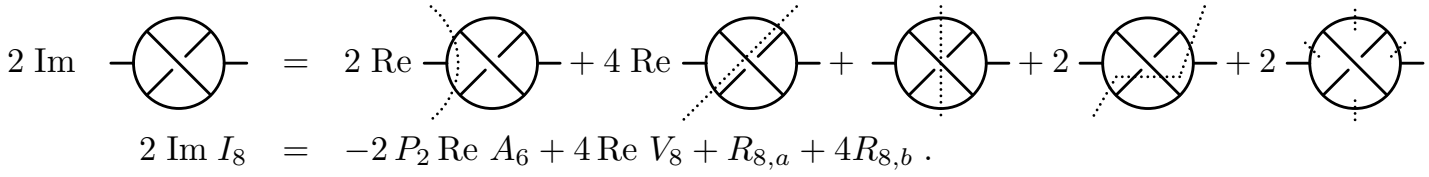

Again, the crossed vertex master integral $A_{6}$ is well known from the literature [44,45] to be

$$
A_{6}=\left(\frac{(4 \pi)^{\epsilon}}{16 \pi^{2}} \frac{\Gamma(1+\epsilon) \Gamma^{2}(1-\epsilon)}{\Gamma(1-2 \epsilon)}\right)^{2}\left(-q^{2}\right)^{-2-2 \epsilon}\left[-\frac{1}{\epsilon^{4}}+\frac{5 \pi^{2}}{6 \epsilon^{2}}+\frac{23}{\epsilon} \zeta_{3}+\frac{103 \pi^{4}}{180}+\mathcal{O}(\epsilon)\right] .
$$

The three-particle cut $V_{8}$ is the integral of the one-loop four-point functions over the three-particle phase space

$$
V_{8}=-i \int \mathrm{d} P S_{3} \frac{1}{s_{12}} \operatorname{Box}\left(s_{13}, s_{13}, s_{12}\right),
$$

with the one-loop box taking the well-known [4] compact form

$$
\operatorname{Box}\left(s_{13}, s_{23}, s_{12}\right)=\left(\frac{(4 \pi)^{\epsilon}}{16 \pi^{2}} \frac{\Gamma(1+\epsilon) \Gamma^{2}(1-\epsilon)}{\Gamma(1-2 \epsilon)}\right)\left(-q^{2}\right)^{-2-\epsilon} \frac{2 i}{\epsilon^{2}} \frac{1}{y_{13} y_{23}}
$$




$$
\begin{aligned}
& {\left[\left(\frac{y_{13} y_{23}}{1-y_{13}}\right)^{-\epsilon}{ }_{2} F_{1}\left(-\epsilon,-\epsilon ; 1-\epsilon ; \frac{1-y_{13}-y_{23}}{1-y_{13}}\right)\right.} \\
& +\left(\frac{y_{13} y_{23}}{1-y_{23}}\right)^{-\epsilon}{ }_{2} F_{1}\left(-\epsilon,-\epsilon ; 1-\epsilon ; \frac{1-y_{13}-y_{23}}{1-y_{23}}\right) \\
& \left.-\left(\frac{y_{13} y_{23}}{\left(1-y_{13}\right)\left(1-y_{23}\right)}\right)^{-\epsilon}{ }_{2} F_{1}\left(-\epsilon,-\epsilon ; 1-\epsilon ; \frac{1-y_{13}-y_{23}}{\left(1-y_{13}\right)\left(1-y_{23}\right)}\right)\right],
\end{aligned}
$$

where in this case $q^{2}=s_{12}+s_{13}+s_{23}$ and $y_{i j}=s_{i j} / q^{2}$. To perform the integration, the three-particle phase space is most conveniently parametrised in terms of the $y_{i j}$ (see Appendix):

$$
\mathrm{d} P S_{3}=(2 \pi)^{-5+4 \epsilon} 2^{-5+2 \epsilon}\left(q^{2}\right)^{1-2 \epsilon} \mathrm{d} \Omega_{3-2 \epsilon} \mathrm{d} \Omega_{2-2 \epsilon}\left(y_{12} y_{13} y_{23}\right)^{-\epsilon} \mathrm{d} y_{12} \mathrm{~d} y_{13} \mathrm{~d} y_{23} \delta\left(1-y_{12}-y_{13}-y_{23}\right) .
$$

Similar integrals were considered in the literature in the context of the integration of subtraction terms appropriate to single unresolved limits of one-loop amplitudes [14 15]. While the first two terms can in fact be straightforwardly integrated over the three-particle phase space, more care is required for the last term. We have used several analytic continuations of hypergeometric functions [38] at intermediate stages where appropriate. Furthermore, some of the integrals could only be carried out on the series representation of hypergeometric functions, the resulting expressions were resummed using Mathematica [46. One finally arrives at

$$
\operatorname{Re} V_{8}=S_{\Gamma}\left(q^{2}\right)^{-2-2 \epsilon}\left[-\frac{5}{2 \epsilon^{4}}+\frac{9 \pi^{2}}{2 \epsilon^{2}}+\frac{89 \zeta_{3}}{\epsilon}+\frac{13 \pi^{4}}{180}+\mathcal{O}(\epsilon)\right] .
$$

As a result, one infers from (4.26):

$$
R_{8, b}=S_{\Gamma}\left(q^{2}\right)^{-2-2 \epsilon}\left[\frac{3}{4 \epsilon^{4}}-\frac{17 \pi^{2}}{12 \epsilon^{2}}-\frac{44 \zeta_{3}}{\epsilon}-\frac{61 \pi^{4}}{60}+\mathcal{O}(\epsilon)\right] .
$$

Finally, we can also employ the unitarity relation to check the correctness of the reduction developed in Section 3 by computing a reducible integral directly through the unitarity relation and comparing it with the result obtained from the reduction procedure. For this exercise, we choose

$$
R_{8, r} \equiv-=\int \mathrm{d} P S_{4} \frac{1}{s_{13} s_{134} s_{24} s_{234}}
$$

which reduces to

$$
\begin{aligned}
R_{8, r} & =\frac{\left(1-7 \epsilon+18 \epsilon^{2}\right)(2-3 \epsilon)(3-4 \epsilon)}{2 \epsilon^{4}} R_{4}+\frac{3(1-2 \epsilon)(1-3 \epsilon)}{2 \epsilon^{2}} R_{6} \\
& =S_{\Gamma}\left(q^{2}\right)^{-2-2 \epsilon}\left[\frac{1}{4 \epsilon^{4}}-\frac{\pi^{2}}{12 \epsilon^{2}}+\frac{7 \zeta_{3}}{\epsilon}+\frac{23 \pi^{4}}{45}+\mathcal{O}(\epsilon)\right] .
\end{aligned}
$$

This integral is related to the imaginary part of 43,28 .

$$
I_{8, r}=-1-\mathcal{O}\left(\epsilon^{0}\right)
$$

for which the unitarity relation reads

$2 \operatorname{Im}$

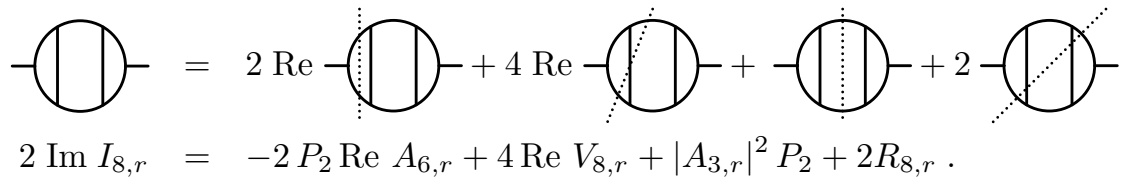


The terms in this relation are easily evaluated as

$$
\begin{aligned}
A_{6, r} & =\left(\frac{(4 \pi)^{\epsilon}}{16 \pi^{2}} \frac{\Gamma(1+\epsilon) \Gamma^{2}(1-\epsilon)}{\Gamma(1-2 \epsilon)}\right)^{2}\left(-q^{2}\right)^{-2-2 \epsilon}\left[-\frac{1}{4 \epsilon^{4}}-\frac{\pi^{2}}{4 \epsilon^{2}}-\frac{6}{\epsilon} \zeta_{3}-\frac{3 \pi^{4}}{20}+\mathcal{O}(\epsilon)\right], \\
\operatorname{Re} V_{8, r} & =S_{\Gamma}\left(q^{2}\right)^{-2-2 \epsilon}\left[-\frac{1}{2 \epsilon^{4}}+\frac{\pi^{2}}{6 \epsilon^{2}}-\frac{5 \zeta_{3}}{\epsilon}-\frac{5 \pi^{4}}{36}+\mathcal{O}(\epsilon)\right], \\
\left|A_{3, r}\right|^{2} & =\left(\frac{(4 \pi)^{\epsilon}}{16 \pi^{2}} \frac{\Gamma(1+\epsilon) \Gamma^{2}(1-\epsilon)}{\Gamma(1-2 \epsilon)}\right)^{2}\left(q^{2}\right)^{-2-2 \epsilon} \frac{1}{\epsilon^{4}},
\end{aligned}
$$

such that together with (4.31), the unitarity relation (4.33) is fulfilled identically. Since only the evaluation of $R_{8, r}$ relied on the reduction of phase space integrals, this provides a strong check on the correct implementation of the reduction algorithm described in Section 3

\section{Numerical Calculation of Master Integrals}

As outlined in 24, the method of sector decomposition [47] to isolate the (infrared) divergences from a given parameter integral and to calculate the resulting pole coefficients numerically can be applied not only to multi-loop integrals 22 23, but also to phase space integrals. To this aim, the phase space for the $1 \rightarrow 4$ particle reaction considered here has to be cast into a convenient parameter integral form, as will be explained below.

The starting point is the expression (A.5) for the four-particle phase space. This form, where the integration variables are the kinematic invariants $s_{i j}$, is most convenient for the sector decomposition algorithm since it is maximally symmetric in the integration variables. In addition, the potential infrared divergences stem a priori only from the limits $s_{i j} \rightarrow 0$ of a certain set of invariants (appearing in the denominator of some matrix element), that is, they are located at the origin of parameter space.

Further, we rescale the Mandelstam invariants, in order to deal with dimensionless parameters, by the definitions

$$
\begin{aligned}
& x_{1}=s_{12} / q^{2}, x_{2}=s_{13} / q^{2}, x_{3}=s_{23} / q^{2}, \\
& x_{4}=s_{14} / q^{2}, x_{5}=s_{24} / q^{2}, x_{6}=s_{34} / q^{2}
\end{aligned}
$$

to arrive at

$$
\int \mathrm{d} P S_{4}=C_{\Gamma}\left(q^{2}\right)^{\frac{3 d}{2}-4} \int\left\{\prod_{j=1}^{6} \mathrm{~d} x_{j} \Theta\left(x_{j}\right)\right\} \delta\left(1-\sum_{i=1}^{6} x_{i}\right)\left[-\lambda\left(x_{1} x_{6}, x_{2} x_{5}, x_{3} x_{4}\right)\right]^{\frac{d-5}{2}} \Theta(-\lambda)
$$

where

$$
C_{\Gamma}=(2 \pi)^{4-3 d} V(d-1) V(d-2) V(d-3) 2^{1-2 d},
$$

$\lambda\left(x_{1} x_{6}, x_{2} x_{5}, x_{3} x_{4}\right)$ is the Källen function already introduced in (4.5) and $V(d)$ is the $d$-dimensional volume defined in (A.2).

After these transformations, we obtain for the integrals $R_{6}$ and $R_{8 a, b}$

$$
\begin{aligned}
R_{6} & =\left(q^{2}\right)^{-2} \int \mathrm{d} P S_{4} \frac{1}{\left(x_{2}+x_{4}+x_{6}\right)\left(x_{3}+x_{5}+x_{6}\right)}, \\
R_{8, a} & =\left(q^{2}\right)^{-4} \int \mathrm{d} P S_{4} \frac{1}{x_{2} x_{3} x_{4} x_{5}}, \\
R_{8, b} & =\left(q^{2}\right)^{-4} \int \mathrm{d} P S_{4} \frac{1}{x_{2} x_{3}\left(x_{2}+x_{4}+x_{6}\right)\left(x_{3}+x_{5}+x_{6}\right)} .
\end{aligned}
$$

The variables in (5.1) are constrained not only by the momentum conserving delta-distribution $\delta\left(1-\sum_{i=1}^{6} x_{i}\right)$, but also by the requirement

$$
\lambda\left(x_{1} x_{6}, x_{2} x_{5}, x_{3} x_{4}\right) \leq 0 \Leftrightarrow x_{1}^{2} x_{6}^{2}+x_{2}^{2} x_{5}^{2}+x_{3}^{2} x_{4}^{2}-2\left(x_{1} x_{3} x_{4} x_{6}+x_{2} x_{3} x_{4} x_{5}+x_{1} x_{2} x_{5} x_{6}\right) \leq 0 .
$$


Therefore the integration variables are hard to factorise and disentangling the overlapping pole structure in general is a highly nontrivial task. That is where the iterated decomposition into sectors until a nonoverlapping form is reached shows its virtues.

\subsection{Sector decomposition}

First we decompose the original integral into a sum of six integrals corresponding to the six "primary sectors" created by inserting unity in the form

$$
1=\sum_{j=1}^{6} \prod_{\substack{k=1 \\ k \neq j}}^{6} \Theta\left(x_{j}-x_{k}\right) .
$$

In each primary sector $j$ created in this way we transform variables according to

$$
x_{k}= \begin{cases}x_{j} t_{k} & \text { if } k \neq j \\ x_{j} & \text { if } k=j\end{cases}
$$

and integrate out $x_{j}$ using the momentum conserving $\delta$-distribution, to arrive at the following form for the phase space integral (where we choose $j=1$ to give a concrete example):

$$
\begin{aligned}
\int \mathrm{d} P S_{4}^{(\sec 1)}= & C_{\Gamma}\left(q^{2}\right)^{\frac{3 d}{2}-4}\left\{\prod_{i=2}^{6} \int \mathrm{d} t_{i} \Theta\left(t_{i}\right) \Theta\left(1-t_{i}\right)\right\} A_{(\sec 1)}^{4-2 d} \\
& {\left[-\lambda^{(\sec 1)}\left(t_{6}, t_{2} t_{5}, t_{3} t_{4}\right)\right]^{\frac{d-5}{2}} \Theta\left(-\lambda^{(\sec 1)}\right), } \\
A_{(\sec 1)}= & 1+\sum_{i=2}^{6} t_{i}, \\
-\lambda^{(\sec 1)}\left(t_{6}, t_{2} t_{5}, t_{3} t_{4}\right)= & t_{6}^{2}+t_{2}^{2} t_{5}^{2}+t_{3}^{2} t_{4}^{2}-2\left(t_{3} t_{4} t_{6}+t_{2} t_{3} t_{4} t_{5}+t_{2} t_{5} t_{6}\right) .
\end{aligned}
$$

Solving the equation $-\lambda^{(\sec 1)}=0$ for $t_{6}$ (or, in general, in sector $j$, for $t_{7-j}$, which occurs simply as $t_{7-j}^{2}$ in $\left.\lambda^{(\operatorname{secj})}\right)$ :

$$
t_{6}^{ \pm}=t_{2} t_{5}+t_{3} t_{4} \pm 2 \sqrt{t_{2} t_{3} t_{4} t_{5}}=\left(\sqrt{t_{2} t_{5}} \pm \sqrt{t_{3} t_{4}}\right)^{2}
$$

and substituting

$$
t_{6}=\tilde{t}_{6}\left(t_{6}^{+}-t_{6}^{-}\right)+t_{6}^{-}=\tilde{t}_{6} 4 \sqrt{t_{2} t_{3} t_{4} t_{5}}+t_{2} t_{5}+t_{3} t_{4}-2 \sqrt{t_{2} t_{3} t_{4} t_{5}},
$$

where now

$$
-\lambda^{(\sec 1)}=\tilde{t}_{6}\left(1-\tilde{t}_{6}\right)\left(t_{6}^{+}-t_{6}^{-}\right)^{2}=16 t_{2} t_{3} t_{4} t_{5} \tilde{t}_{6}\left(1-\tilde{t}_{6}\right)
$$

leads to

$$
\begin{aligned}
\int \mathrm{d} P S_{4}^{(\sec 1)=} & S_{\Gamma}\left(q^{2}\right)^{d-2} \frac{\Gamma(1-2 \epsilon)}{\Gamma^{2}\left(\frac{1}{2}-\epsilon\right)}\left\{\prod_{i=2}^{5} \int \mathrm{d} t_{i} \Theta\left(t_{i}\right) \Theta\left(1-t_{i}\right)\right\}\left[t_{2} t_{3} t_{4} t_{5}\right]^{-\epsilon} \\
& \int \mathrm{d} \tilde{t}_{6}\left[\tilde{t}_{6}\left(1-\tilde{t}_{6}\right)\right]^{-\frac{1}{2}-\epsilon} \Theta\left(\tilde{t}_{6}\right) \Theta\left(1-\left[\tilde{t}_{6}\left(t_{6}^{+}-t_{6}^{-}\right)+t_{6}^{-}\right]\right) A_{(\text {sec } 1)}^{-4+4 \epsilon} .
\end{aligned}
$$

However, as a consequence of the transformation (5.8), singularities at $t_{i}=1$ can also occur now. For example, a factor $1 / t_{6}$ that might be present in the matrix element can become singular for $\tilde{t}_{6} \rightarrow 0, t_{i=2,3,4,5} \rightarrow 1$. As the subtractions performed later rely on the fact that only the limits $t_{i} \rightarrow 0$ can lead to a singularity, these potential singularities are remapped to the origin in the following way: The program checks if a (non-empty) set $\mathcal{T}$ exists such that a denominator vanishes if all $t_{i} \in \mathcal{T}$ are one and $\tilde{t}_{k} \rightarrow 0$. For the $t_{i} \in \mathcal{T}$, it is checked if in addition $t_{i} \rightarrow 0$ is singular. If yes, the integration region is split according to $\int_{0}^{1} d t_{i}=\int_{0}^{\frac{1}{2}} d t_{i}+\int_{\frac{1}{2}}^{1} d t_{i}$ and 
the resulting integrals are remapped to integrals from zero to one. Otherwise, simply the transformations $t_{i} \rightarrow 1-t_{i}$ are performed for the $t_{i} \in \mathcal{T}$. Note that the limits $\left\{\tilde{t}_{k} \rightarrow 0, t_{i} \rightarrow 1\left(t_{i} \in \mathcal{T}\right)\right\}$ do in most cases not lead to a real $1 / \epsilon$ singularity upon integration, even if they make the denominator vanish, but they will be remapped nevertheless for the sake of numerical stability.

These transformations as well as the sector decomposition are iterated if necessary, that is, if a (composite $^{1}$ ) denominator of the integrand still can vanish at a certain phase space point.

The iterated sector decomposition proceeds as follows: The program determines the minimal set $\mathcal{S}=$ $\left\{t_{\alpha_{1}}, \ldots, t_{\alpha_{r}}\right\}$ of parameters which in the limit $t_{\alpha} \rightarrow 0$ make a composite denominator of the matrix element vanish. (The parameters $\tilde{t}$ have been renamed $t$ ). Then the corresponding integrals are decomposed into $r$ subsectors according to

$$
\prod_{i=1}^{r} \Theta\left(1-t_{\alpha_{i}}\right)=\sum_{k=1}^{r} \prod_{\substack{i=1 \\ i \neq k}}^{r} \Theta\left(t_{\alpha_{k}}-t_{\alpha_{i}}\right) .
$$

In each new subsector, the variables are remapped to the unit cube by the substitution

$$
t_{\alpha_{i}} \rightarrow \begin{cases}t_{\alpha_{k}} t_{\alpha_{i}} & \text { for } \quad i \neq k \\ t_{\alpha_{k}} & \text { for } \quad i=k\end{cases}
$$

By construction, $t_{\alpha_{k}}$ factorises from at least one of the denominator functions. The sector decomposition procedure is iterated until the denominators are of the form $D=1+f\left(t_{\alpha}\right)$, where $f\left(t_{\alpha}\right)$ is a non-negative function of the parameters $t_{\alpha}$. Then subtractions of the singularities, which are now all factorised explicitly, are carried out, using identities like

$$
\int_{0}^{1} \mathrm{~d} t_{\alpha} t_{\alpha}^{-1-\kappa \epsilon} \mathcal{F}\left(t_{\alpha}, t_{\beta \neq \alpha}\right)=-\frac{1}{\kappa \epsilon} \mathcal{F}\left(0, t_{\beta \neq \alpha}\right)+\int_{0}^{1} \mathrm{~d} t_{\alpha} t_{\alpha}^{-1-\kappa \epsilon}\left\{\mathcal{F}\left(t_{\alpha}, t_{\beta \neq \alpha}\right)-\mathcal{F}\left(0, t_{\beta \neq \alpha}\right)\right\}
$$

where $\lim _{t_{\alpha} \rightarrow 0} \mathcal{F}\left(t_{\alpha}, t_{\beta \neq \alpha}\right)$ is finite by construction.

After having carried out the subtractions for each $t_{\alpha}$, the expansion in $\epsilon$ can be performed. The result is a Laurent series in $\epsilon$ where the pole coefficients are finite functions of the parameters $t_{\alpha}$ which can be integrated numerically.

Note that the coefficient functions of a certain pole, produced by the iterated decomposition of a certain primary sector into subsectors, are all summed over before numerical integration in order to avoid large numerical errors due to large cancellations. In contrast, the contributions from the primary sectors (i.e. six contributions in the $1 \rightarrow 4$ case) are summed over only after the numerical integration. In this way, one has a strong check at hand if for topological reasons (symmetry of the considered integral) some primary sectors have to yield identical results, while the numerical error is not substantially increased.

We would like to emphasize that the functions occurring in the phase space integrals are quite different from the functions present in any virtual multi-loop integral after Feynman parametrisation: They are not polynomial anymore, as they unavoidably contain square-roots (and fake singularities away from the origin, as discussed above). This is of course not a principal problem for the algorithm, but has to be dealt with appropriately.

The $\Theta$-function and the $A_{(s e c j)}$ term in (5.9) become quite involved expressions after several iterations, but do not present any problem for the numerical integration.

The program consists of 3 building blocks: The first one serves to perform the iterated sector decomposition until a form of the integrand is reached where all singularities are factored out explicitly as described above. The corresponding algorithm has been implemented in Mathematica [4].

Then the subtractions and the expansion in $\epsilon$ are carried out to obtain a set of finite functions for each pole coefficient. These functions are then translated into FORTRAN functions. Up to this stage, the method is not "numerical" yet. Only the complicated structure and the large number of these functions hampers their analytical integration.

\footnotetext{
${ }^{1}$ By "composite" we mean that the denominator is a nontrivial function of parameters $t_{i}$, as for example $\left(t_{2}+t_{4}+t_{6}\right)$ present in $R_{8, b}$, and not only a trivial factor. Note that after the transformation (5.8) a previously trivial factor like $1 / t_{6}$ becomes composite.
} 
The third part consists in the numerical integration of these functions, where the Monte Carlo program BASES [48 is used. All FORTRAN files are generated automatically using Maple [37 and perl. Perl routines also serve to automate compilation, job submission and summation of the results from the different primary sectors, such that the only "manual" input is the matrix element and the desired expansion level in $\epsilon$.

\section{$5.2 \quad$ Numerical Results}

The program produces an overall number of 13 subsectors for the integral $R_{6}, 690$ subsectors for $R_{8, a}$ and 321 subsectors for $R_{8, b}$. The integration time ranges from about 10 minutes for $R_{6}$ to about 24 hours for $R_{8, a}$ if a numerical accuracy better than $1 \%$ is demanded.

The following numerical results are obtained for the master integrals:

$$
\begin{aligned}
R_{4} & =S_{\Gamma}\left(q^{2}\right)^{2-2 \epsilon}\left[0.08335+0.81946 \epsilon+4.1413 \epsilon^{2}+14.398 \epsilon^{3}+38.880 \epsilon^{4}+\mathcal{O}\left(\epsilon^{5}\right)\right], \\
R_{6} & =S_{\Gamma}\left(q^{2}\right)^{-2 \epsilon}\left[0.64498+7.0423 \epsilon+40.507 \epsilon^{2}+\mathcal{O}\left(\epsilon^{3}\right)\right] \\
R_{8, a} & =S_{\Gamma}\left(q^{2}\right)^{-2-2 \epsilon}\left[\frac{5.0003}{\epsilon^{4}}-\frac{0.0013}{\epsilon^{3}}-\frac{65.832}{\epsilon^{2}}-\frac{151.53}{\epsilon}+37.552+\mathcal{O}(\epsilon)\right], \\
R_{8, b} & =S_{\Gamma}\left(q^{2}\right)^{-2-2 \epsilon}\left[\frac{0.74986}{\epsilon^{4}}-\frac{0.00009}{\epsilon^{3}}-\frac{14.001}{\epsilon^{2}}-\frac{52.911}{\epsilon}-99.031+\mathcal{O}(\epsilon)\right] .
\end{aligned}
$$

The reducible integral $R_{8, r}$ also has been calculated numerically to complete the cross-checks:

$$
R_{8, r}=S_{\Gamma}\left(q^{2}\right)^{-2-2 \epsilon}\left[\frac{0.24999}{\epsilon^{4}}-\frac{0.00025}{\epsilon^{3}}-\frac{0.82251}{\epsilon^{2}}+\frac{8.4106}{\epsilon}+49.748+\mathcal{O}(\epsilon)\right] .
$$

The agreement of the numerical and analytical results is better than $1 \%$.

\section{Summary and Conclusions}

In this paper, we studied the inclusive four-particle phase space integrals of $(1 \rightarrow 4)$-parton QCD matrix elements. These integrals contain infrared divergences due to the soft or collinear emission of up to two partons, which occur also in NNLO calculations of jet observables. We demonstrated that these phase space integrals can be expressed as a linear combination of only four master integrals. These four master integrals were computed both analytically and numerically up to their fourth order terms in dimensional regularisation, as required for NNLO calculations. The analytic results for them are:

$$
\begin{aligned}
& R_{4}=S_{\Gamma}\left(q^{2}\right)^{2-2 \epsilon} \frac{\Gamma^{5}(1-\epsilon) \Gamma(2-2 \epsilon)}{\Gamma(3-3 \epsilon) \Gamma(4-4 \epsilon)} \\
& R_{6}=S_{\Gamma}\left(q^{2}\right)^{-2 \epsilon}\left[-1+\frac{\pi^{2}}{6}+\epsilon\left(-12+\frac{5 \pi^{2}}{6}+9 \zeta_{3}\right)\right. \\
&\left.+\epsilon^{2}\left(-91+\frac{9 \pi^{2}}{2}+45 \zeta_{3}+\frac{61 \pi^{4}}{180}\right)+\mathcal{O}\left(\epsilon^{3}\right)\right], \\
& R_{8, a}=S_{\Gamma}\left(q^{2}\right)^{-2-2 \epsilon}\left[\frac{5}{\epsilon^{4}}-\frac{20 \pi^{2}}{3 \epsilon^{2}}-\frac{126 \zeta_{3}}{\epsilon}+\frac{7 \pi^{4}}{18}+\mathcal{O}(\epsilon)\right], \\
& R_{8, b=}=S_{\Gamma}\left(q^{2}\right)^{-2-2 \epsilon}\left[\frac{3}{4 \epsilon^{4}}-\frac{17 \pi^{2}}{12 \epsilon^{2}}-\frac{44 \zeta_{3}}{\epsilon}-\frac{61 \pi^{4}}{60}+\mathcal{O}(\epsilon)\right] .
\end{aligned}
$$

The inclusive four-particle phase space integrals of QCD $1 \rightarrow 4$ matrix elements may in future work be used for the construction of infrared subtraction terms for the real contributions to NNLO jet calculations. In the case of two-jet production in $e^{+} e^{-}$collisions, the $1 \rightarrow 4$ matrix elements can serve immediately as subtraction terms, since the only requirements on subtraction terms are that they coincide with the full 
matrix element in all infrared singular limits (which is clearly the case) and that they can be integrated analytically over the inclusive phase space (which we have demonstrated here). Constructing NNLO subtraction terms for the double real emission contributions to three-jet production in $e^{+} e^{-}$collisions from $1 \rightarrow 4$ matrix elements, which can serve to locally approximate the full $1 \rightarrow 5$ matrix element in certain limits, is a yet outstanding task. To the same aim, one could also look for a way to relate already existing NNLO subtraction terms [16, 17, 18 to $1 \rightarrow 4$ QCD matrix elements. In this context, it should be pointed out that the frequently used NLO dipole subtraction terms [12 for multi-jet production in $e^{+} e^{-}$ annihilation can in fact be recombined to yield $1 \rightarrow 3 \mathrm{QCD}$ matrix elements.

In the course of the calculations presented in this paper, new analytical and numerical methods for handling infrared divergent phase space integrals were developed. In particular, the tripole parametrisation of the four-particle phase space incorporates explicitly the phase space factorisation from an $(n+2)$-parton phase space to an $n$-parton phase space and a factor which accounts for the singular emission, as required for $n$-jet production at NNLO. This tripole formulation has the advantage that it avoids the introduction of non-linear transformations of momenta, which is crucial for the analytic integration. For the construction of an NNLO Monte Carlo parton-level event generator, a smooth mapping of all potentially singular regions of phase space is essential for numerical stability. Such a mapping can for example be achieved using nonlinear momentum transformations [17, 18. However, the phase space parametrisations used to integrate the subtraction terms analytically and those used in the Monte Carlo program do not need to be the same.

Formulating the unitarity relations between phase space and loop integrals in a form which connects different master integrals enabled us to deduce three of the four master integrals from known multi-loop integrals, such that only one of them had to be calculated explicitly. Thus we were able to use multi-loop techniques in order to obtain results for complicated phase space integrals.

The iterated sector decomposition, a method originally developed for infrared divergent loop integrals, where the poles are isolated automatically and their coefficients are calculated numerically, was extended here to phase space integrals. This technique is particularly powerful for $(1 \rightarrow n)$-particle reactions, where the phase space integrals depend only on one overall scale. As a consequence, the pole coefficients are just numbers which can be calculated numerically once and for all. This method can also be useful in cases where an analytic calculation of the phase space integrals cannot be achieved anymore, not only for inclusive cross sections, but also in view of the construction of an NNLO Monte Carlo program, where experimental cuts acting on the phase space boundaries can complicate the analytic integrations.

The excellent agreement between analytical and numerical results for the four master integrals evaluated here provides a strong check on both calculational approaches.

\section{Acknowledgements}

AG and TG wish to thank Rolf Mertig for numerous instructive discussions on hypergeometric functions and their usage within the computer algebra system Mathematica. AG acknowledges the kind hospitality of the Institut für Theoretische Physik of RWTH Aachen, where part of this work was performed. GH would like to thank the University of Zürich for its kind hospitality. This work was supported by the Swiss National Funds (SNF) under contract 200021-101874.

\section{A Massless Multi-parton Phase Space}

The $n$-particle phase space in the $(1 \rightarrow n)$-particle decay reaction kinematics $\left(q \rightarrow p_{1}+\ldots+p_{n}\right)$ reads in dimensional regularisation with $d=4-2 \epsilon$ space-time dimensions

$$
\mathrm{d} P S_{n}=\frac{\mathrm{d}^{d-1} p_{1}}{2 E_{1}(2 \pi)^{d-1}} \cdots \frac{\mathrm{d}^{d-1} p_{n}}{2 E_{n}(2 \pi)^{d-1}}(2 \pi)^{d} \delta^{d}\left(q-p_{1}-\ldots-p_{n}\right) .
$$

In the present work, we are in particular concerned about the two-, three- and four-parton phase space.

In practical applications, it turns out that the above expression for the multi-particle phase space in terms of momenta is not convenient. We therefore use expressions in terms of kinematic invariants 
$s_{i j}=2 p_{i} \cdot p_{j}$ and angular volumes instead. Further, we factorise out overall rotations of the coordinate frame in $d$ dimensions into the $d$-dimensional hypersphere $\mathrm{d} \Omega_{d}$ with volume

$$
V(d) \equiv \int \mathrm{d} \Omega_{d}=\frac{2 \pi^{d / 2}}{\Gamma(d / 2)}
$$

The two-particle phase space in these variables simply reads

$$
\mathrm{d} P S_{2}=(2 \pi)^{2-d}\left(s_{12}\right)^{\frac{d-4}{2}} \frac{\mathrm{d} \Omega_{d-1}}{2^{d-1}} \mathrm{~d} s_{12} \delta\left(q^{2}-s_{12}\right) .
$$

For the three-particle phase space, one finds

$$
\mathrm{d} P S_{3}=(2 \pi)^{3-2 d} 2^{-1-d}\left(q^{2}\right)^{\frac{2-d}{2}} \mathrm{~d} \Omega_{d-1} \mathrm{~d} \Omega_{d-2}\left(s_{12} s_{13} s_{23}\right)^{\frac{d-4}{2}} \mathrm{~d} s_{12} \mathrm{~d} s_{13} \mathrm{~d} s_{23} \delta\left(q^{2}-s_{12}-s_{13}-s_{23}\right) .
$$

Finally, the four-particle phase space is

$$
\begin{aligned}
\mathrm{d} P S_{4}= & (2 \pi)^{4-3 d}\left(q^{2}\right)^{3-\frac{d}{2}} 2^{1-2 d}\left(-\Delta_{4}\right)^{\frac{d-5}{2}} \Theta\left(-\Delta_{4}\right) \delta\left(q^{2}-s_{12}-s_{13}-s_{14}-s_{23}-s_{24}-s_{34}\right) \\
& \mathrm{d} \Omega_{d-1} \mathrm{~d} \Omega_{d-2} \mathrm{~d} \Omega_{d-3} \mathrm{~d} s_{12} \mathrm{~d} s_{13} \mathrm{~d} s_{14} \mathrm{~d} s_{23} \mathrm{~d} s_{24} \mathrm{~d} s_{34},
\end{aligned}
$$

where the Gram determinant $\Delta_{4}$ is given by

$$
+\Delta_{4}=\lambda\left(s_{12} s_{34}, s_{13} s_{24}, s_{14} s_{23}\right), \lambda(x, y, z)=x^{2}+y^{2}+z^{2}-2(x y+x z+y z) .
$$

In several parts of this paper, we also need the total $n$-particle phase space volume

$$
P_{n}=\int \mathrm{d} P S_{n}
$$

Using the unitarity relation of Section 4.3 we find a compact expression for arbitrary $n$ :

$$
P_{n}=2^{5-4 n-2 \epsilon+2 n \epsilon} \pi^{3-2 n-\epsilon+n \epsilon} \frac{\Gamma^{n}(1-\epsilon)}{\Gamma((n-1)(1-\epsilon)) \Gamma(n(1-\epsilon))}\left(q^{2}\right)^{n-2+\epsilon-n \epsilon},
$$

in particular:

$$
\begin{aligned}
& P_{2}=2^{-3+2 \epsilon} \pi^{-1+\epsilon} \frac{\Gamma(1-\epsilon)}{\Gamma(2-2 \epsilon)}\left(q^{2}\right)^{-\epsilon}, \\
& P_{3}=2^{-7+4 \epsilon} \pi^{-3+2 \epsilon} \frac{\Gamma^{3}(1-\epsilon)}{\Gamma(2-2 \epsilon) \Gamma(3-3 \epsilon)}\left(q^{2}\right)^{1-2 \epsilon}, \\
& P_{4}=2^{-11+6 \epsilon} \pi^{-5+3 \epsilon} \frac{\Gamma^{4}(1-\epsilon)}{\Gamma(3-3 \epsilon) \Gamma(4-4 \epsilon)}\left(q^{2}\right)^{2-3 \epsilon} .
\end{aligned}
$$

\section{References}

[1] Z. Bern, L.J. Dixon and A. Ghinculov, Phys. Rev. D 63 (2001) 053007 hep-ph/0010075.

[2] C. Anastasiou, E.W.N. Glover, C. Oleari and M.E. Tejeda-Yeomans, Nucl. Phys. B 601 (2001) 318 hep-ph/0010212; 601 (2001) 347 hep-ph/0011094; 605 (2001) 486 hep-ph/0101304;

E.W.N. Glover, C. Oleari and M.E. Tejeda-Yeomans, Nucl. Phys. 605 (2001) 467 hep-ph/0102201; C. Anastasiou, E.W.N. Glover and M.E. Tejeda-Yeomans, Nucl. Phys. B 629 (2002) 255 hep-ph/0201274;

E.W.N. Glover and M.E. Tejeda-Yeomans, JHEP 0306 (2003) 033 hep-ph/0304169.

[3] Z. Bern, A. De Freitas and L.J. Dixon, JHEP 0109 (2001) 037 hep-ph/0109078; JHEP 0203 (2002) 018 hep-ph/0201161; JHEP 0306 (2003) 028 hep-ph/0304168. 
[4] Z. Bern, A. De Freitas, L.J. Dixon, A. Ghinculov and H.L. Wong, JHEP 0111 (2001) 031 hep-ph/0109079;

T. Binoth, E.W.N. Glover, P. Marquard and J.J. van der Bij, JHEP 0205 (2002) 060 hep-ph/0202266.

[5] L.W. Garland, T. Gehrmann, E.W.N. Glover, A. Koukoutsakis and E. Remiddi, Nucl. Phys. B 627 (2002) 107 hep-ph/0112081 and 642 (2002) 227 hep-ph/0206067.

[6] S. Moch, P. Uwer and S. Weinzierl, Phys. Rev. D 66 (2002) 114001 hep-ph/0207043.

[7] T. Gehrmann and E. Remiddi, Nucl. Phys. B 640 (2002) 379 hep-ph/0207020.

[8] L.J. Dixon and A. Signer, Phys. Rev. Lett. 78 (1997) 811 hep-ph/9609460; Phys. Rev. D 56 (1997) 4031 hep-ph/9706285;

Z. Nagy and Z. Trocsanyi, Phys. Rev. Lett. 79 (1997) 3604 hep-ph/9707309;

J. Campbell, M.A. Cullen and E.W.N. Glover, Eur. Phys. J. C 9 (1999) 245 hep-ph/9809429;

S. Weinzierl and D.A. Kosower, Phys. Rev. D 60 (1999) 054028 hep-ph/9901277.

[9] Z. Nagy and Z. Trocsanyi, Phys. Rev. Lett. 87 (2001) 082001 hep-ph/0104315;

Z. Nagy, Phys. Rev. Lett. 88 (2002) 122003 hep-ph/0110315; hep-ph/0307268

J. Campbell and R.K. Ellis, Phys. Rev. D 65 (2002) 113007 hep-ph/0202176.

[10] Z. Kunszt and D.E. Soper, Phys. Rev. D 46 (1992) 192;

S.D. Ellis, Z. Kunszt and D.E. Soper, Phys. Rev. Lett. 69 (1992) 3615 hep-ph/9208249.

[11] W.T. Giele and E.W.N. Glover, Phys. Rev. D 46 (1992) 1980.

[12] S. Catani and M.H. Seymour, Nucl. Phys. B 485 (1997) 291 [Erratum-ibid. B 510 (1997) 503] hep-ph/9605323.

[13] Z. Bern, L.J. Dixon, D.C. Dunbar and D.A. Kosower, Nucl. Phys. B 425 (1994) 217 hep-ph/9403226;

D.A. Kosower, Nucl. Phys. B 552 (1999) 319 hep-ph/9901201;

D.A. Kosower and P. Uwer, Nucl. Phys. B 563 (1999) 477 hep-ph/9903515;

Z. Bern, V. Del Duca and C.R. Schmidt, Phys. Lett. B 445 (1998) 168 hep-ph/9810409;

Z. Bern, V. Del Duca, W.B. Kilgore and C.R. Schmidt, Phys. Rev. D 60 (1999) 116001 hep-ph/9903516;

S. Catani and M. Grazzini, Nucl. Phys. B 591 (2000) 435 hep-ph/0007142.

[14] D.A. Kosower, Phys. Rev. Lett. 91 (2003) 061602 hep-ph/0301069.

[15] S. Weinzierl, JHEP 0307 (2003) 052 hep-ph/0306248.

[16] J. Campbell and E.W.N. Glover, Nucl. Phys. B 527 (1998) 264 hep-ph/9710255;

S. Catani and M. Grazzini, Phys. Lett. B 446 (1999) 143 hep-ph/9810389; Nucl. Phys. B 570 (2000) 287 hep-ph/9908523;

F.A. Berends and W.T. Giele, Nucl. Phys. B 313 (1989) 595.

[17] D.A. Kosower, Phys. Rev. D 67 (2003) 116003 hep-ph/0212097.

[18] S. Weinzierl, JHEP 0303 (2003) 062 hep-ph/0302180.

[19] A. Gehrmann-De Ridder, T. Gehrmann and E.W.N. Glover, Phys. Lett. B 414 (1997) 354 hep-ph/9705305.

[20] A. Gehrmann-De Ridder and E.W.N. Glover, Nucl. Phys. B 517 (1998) 269 hep-ph/9707224.

[21] D.A. Kosower and P. Uwer, hep-ph/0307031

[22] T. Binoth and G. Heinrich, Nucl. Phys. B 585 (2000) 741 hep-ph/0004013.

[23] T. Binoth and G. Heinrich, hep-ph/0305234 
[24] G. Heinrich, Nucl. Phys. Proc. Suppl. 116 (2003) 368 hep-ph/0211144.

[25] R.E. Cutkosky, J. Math. Phys. 1 (1960) 429.

[26] C. Anastasiou and K. Melnikov, Nucl. Phys. B 646 (2002) 220 hep-ph/0207004.

[27] F.V. Tkachov, Phys. Lett. 100B (1981) 65.

[28] K.G. Chetyrkin and F.V. Tkachov, Nucl. Phys. B192 (1981) 159.

[29] S.G. Gorishnii, S.A. Larin, L.R. Surguladze and F.V. Tkachov, Comput. Phys. Comm. 55 (1989) 381; S.A. Larin, F.V. Tkachov and J.A.M. Vermaseren, NIKHEF-H-91-18.

[30] J.A.M. Vermaseren, Symbolic Manipulation with FORM, Version 2, CAN, Amsterdam, 1991 and New features of FORM, math-ph/0010025

[31] R. Harlander and M. Steinhauser, Prog. Part. Nucl. Phys. 43 (1999) 167 hep-ph/9812357.

[32] M. Steinhauser, Phys. Rept. 364 (2002) 247 hep-ph/0201075.

[33] P.A. Baikov and V.A. Smirnov, Phys. Lett. B 477 (2000) 367 hep-ph/0001192.

[34] R. Harlander, Phys. Lett. B 492 (2000) 74 hep-ph/0007289.

[35] S. Laporta, Int. J. Mod. Phys. A 15 (2000) 5087 hep-ph/0102033.

[36] T. Gehrmann and E. Remiddi, Nucl. Phys. B 580 (2000) 485 hep-ph/9912329.

[37] Maple 7, Copyright 2001 by Waterloo Maple Software and the University of Waterloo.

[38] A. Erdélyi (ed.), Higher Transcendental Functions, vol. 1, McGraw-Hill (New York, 1953);

A. Erdélyi (ed.), Tables of Integral Transforms, vol. 2, McGraw-Hill (New York, 1954).

[39] A. Gehrmann-De Ridder, Ph.D. thesis, University of Durham (1997).

[40] Z. Bern, L.J. Dixon, D.C. Dunbar and D.A. Kosower, Nucl. Phys. B 435 (1995) 59 hep-ph/9409265; Z. Bern, L.J. Dixon and D.A. Kosower, Nucl. Phys. Proc. Suppl. 51C (1996) 243 hep-ph/9606378.

[41] A. Bassetto, G. Heinrich, Z. Kunszt and W. Vogelsang, Phys. Rev. D 58 (1998) 094020 hep-ph/9805283.

[42] D.E. Soper, Phys. Rev. Lett. 81 (1998) 2638 hep-ph/9804454; Phys. Rev. D 62 (2000) 014009 hep-ph/9910292.

[43] K.G. Chetyrkin, A.L. Kataev and F.V. Tkachov, Nucl. Phys. B 174 (1980) 345.

[44] G. Kramer and B. Lampe, J. Math. Phys. 28 (1987) 945.

[45] V.A. Smirnov, Phys. Lett. B 460 (1999) 397 hep-ph/9905323;

V.A. Smirnov and O.L. Veretin, Nucl. Phys. B 566 (2000) 469 hep-ph/9907385;

J.B. Tausk, Phys. Lett. B 469 (1999) 225 hep-ph/9909506;

C. Anastasiou, T. Gehrmann, C. Oleari, E. Remiddi and J.B. Tausk, Nucl. Phys. B 580 (2000) 577 hep-ph/0003261;

T. Gehrmann and E. Remiddi, Nucl. Phys. B (Proc. Suppl.) 89 (2000) 251 hep-ph/0005232;

C. Anastasiou, J.B. Tausk and M.E. Tejeda-Yeomans, Nucl. Phys. B (Proc. Suppl.) 89 (2000) 262 hep-ph/0005328;

T. Gehrmann and E. Remiddi, Nucl. Phys. B 601 (2001) 248 hep-ph/0008287; 601 (2001) 287 hep-ph/0101124.

[46] MATHEMATICA 5.0, Copyright 2003 by Wolfram Research. 
[47] K. Hepp, Commun. Math. Phys. 2 (1966) 301;

M. Roth and A. Denner, Nucl. Phys. B 479 (1996) 495 hep-ph/9605420.

[48] S. Kawabata, Comp. Phys. Comm. 88 (1995) 309. 